\title{
Globe
}

Revue internationale d'études québécoises

\section{La reconnaissance de la langue des signes québécoise (LSQ) pour le développement de la littératie et de la participation sociale de la personne sourde au Québec Recognition of Quebec Sign Language (LSQ) and the Development of Deaf Literacy and Social Participation in Quebec}

\author{
Sylvain Letscher, Ghyslain Parent et Rollande Deslandes
}

Volume 16, numéro 1, 2013

La littératie dans les études québécoises

URI : https://id.erudit.org/iderudit/1018177ar

DOI : https://doi.org/10.7202/1018177ar

Aller au sommaire du numéro

Éditeur(s)

Globe, Revue internationale d'études québécoises

ISSN

1481-5869 (imprimé)

1923-8231 (numérique)

Découvrir la revue

Citer cet article

Letscher, S., Parent, G. \& Deslandes, R. (2013). La reconnaissance de la langue des signes québécoise (LSQ) pour le développement de la littératie et de la participation sociale de la personne sourde au Québec. Globe, 16(1), 45-70. https://doi.org/10.7202/1018177ar
Résumé de l'article

Le but de cet article est de relever la manière dont, au Québec, les familles, l'école et la communauté facilitent le développement de la participation sociale de personnes sourdes gestuelles ou bilingues au moyen de la littératie. L'étude renvoie au modèle des obstacles et des facilitateurs au développement de la participation sociale de Letscher, Parent et Deslandes (2009). La méthodologie s'appuie sur l'étude de cas qualitative auprès de 22 répondants, dont sept personnes sourdes, leurs mères, pères et enseignants. Les résultats illustrent un contexte d'organisation des services marqué par la difficulté à reconnaître la langue des signes québécoise (LSO) comme première langue d'apprentissage. Les personnes sourdes présentent un profil de résignation, d'oppression, de résilience ou de libération dans les domaines de l'éducation et du travail, qui prend forme dans le développement de la participation sociale et de la littératie de la personne sourde. 


\title{
LA RECONNAISSANCE DE LA LANGUE DES SIGNES QUÉBÉCOISE (LSQ) POUR LE DÉVELOPPEMENT DE LA LITTÉRATIE ET DE LA PARTICIPATION SOCIALE DE LA PERSONNE SOURDE AU QUÉBEC
}

\author{
SYLVAIN LETSCHER \\ Université du Québec à Trois-Rivières \\ GHYSLAIN PARENT \\ Université du Québec à Trois-Rivières
}

ROLLANDE DESLANDES

Université du Québec à Trois-Rivières

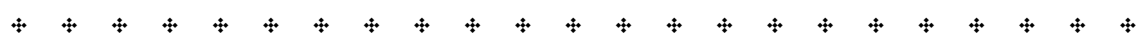

Résumé - Le but de cet article est de relever la manière dont, au Québec, les familles, l'école et la communauté facilitent le développement de la participation sociale de personnes sourdes gestuelles ou bilingues au moyen de la littératie. L'étude renvoie au modèle des obstacles et des facilitateurs au développement de la participation sociale de Letscher, Parent et Deslandes (2009). La méthodologie s'appuie sur l'étude de cas qualitative auprès de 22 répondants, dont sept personnes sourdes, leurs mères, pères et enseignants. Les résultats illustrent un contexte d'organisation des services marqué par la difficulté à reconnaître la langue des signes québécoise (LSQ) comme première langue d'apprentissage. Les personnes sourdes présentent un profil de résignation, d'oppression, de résilience ou de libération dans les domaines de l'éducation et du travail, qui prend forme dans le développement de la participation sociale et de la littératie de la personne sourde. 


\section{Recognition of Quebec Sign Language (LSQ)}

\section{and the Development of Deaf Literacy and Social Participation in Quebec}

Abstract - The aim of this paper is to understand how families, schools, and communities use literacy to facilitate and encourage the social participation of signing deaf persons in Quebec. The study refers to the model of barriers and facilitators in social participation developed by Letscher, Parent, and Deslandes (2009). Methodologically, it is based on a qualitative case study involving twenty-two participants, seven of whom were deaf. The other participants were parents or influential teachers. The results of the study illustrate how the difficulty of having Quebec Sign Language (LSQ) recognized as a primary language of instruction impacts the organization of services. Through the development of social participation and literacy, the deaf participants variously adopted an outlook of resignation, oppression, resilience, or liberation in the fields of education and work.

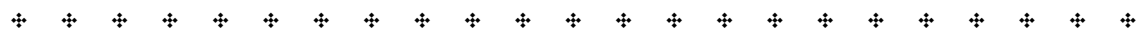

Au Québec, de 1834 jusqu'en 1960 environ, les Sourds sont regroupés dans des institutions spécialisées où l'enseignement s'effectue en signes et non en langue des signes ${ }^{1}$. Les tenants de l' " oralisme» n'ayant pas réussi à résoudre les difficultés de communication des enfants sourds, les institutions proposent, dans les années 1960, l'approche de la "communication totale». Elle autorise l'utilisation de tous les médias disponibles, y compris la langue des signes, mais celle-ci n'est toutefois que rarement utilisée $^{2}$. L'approche oraliste vise l'apprentissage premier de la langue majoritaire à partir d'un ou de plusieurs médias de communication, dont l'audition résiduelle et son amplification, la lecture labiale, le langage parlé complété et le français signé ${ }^{3}$.

Les travaux de William Stokoe, à partir de $1960^{4}$, permettront de reconnaître que la langue des signes répond à tous les critères définissant une langue, en dehors de celui du caractère verbal, puisqu'elle met en jeu le feedback, un lexique et une grammaire ${ }^{5}$. Les années 1970 donnent naissance au différentialisme sourd avec l'américanisation des revendications identitaires sourdes (une histoire portée par des tensions sociales) alimentées par

$$
4+4
$$

1. Colette Dubuisson et Rachel BerThiaume, Entre parents, parlons surdité, Montréal, Université du Québec à Montréal, Groupe de recherche sur la LSQ et le bilinguisme sourd, 2005, p. 34.

2. Ibid, p. 30-31.

3. Marguerite BLAIS, Variantes sur la culture sourde: quêtes identitaires au cour de la communication, Montréal, Université du Québec à Montréal, 2005, p. 95.

4. Ibid, p. 86.

5. Marie-Hélène Herzog, Psychomotricité, relaxation et surdité, Paris, Masson, 1995, p. 74. 
les philosophies d'enseignement et de traitement des enfants sourds, qui explicitent les logiques de revendications identitaires contemporaines des Sourds au Québec ${ }^{6}$. Le substantif «Sourd» avec un $S$ majuscule fait référence à la personne sourde qui utilise la langue des signes et se considère comme appartenant à la culture sourde ${ }^{7}$.

La culture sourde est constituée autour de cinq critères sociologiques, qui sont la langue, les valeurs, les traditions, les normes et l'identité ${ }^{8}$. Elle privilégie l'utilisation de la langue des signes ${ }^{9}$. Les valeurs propres à la culture sourde renvoient, pour les Sourds, au fait de se regrouper entre eux pour leurs loisirs et la défense de leurs droits ${ }^{10}$. Les traditions se rapportent à l'histoire des Sourds ${ }^{11}$. Les normes réferent aux règles de comportement dans la communauté sourde ${ }^{12}$. Une autre composante est le fait de s'identifier à la culture des personnes sourdes ${ }^{13}$.

Au milieu des années 1970, le rapport du Comité provincial de l'enfance exceptionnelle (COPEX) ${ }^{14}{ }^{\prime}$ 'inquiète du fait que les enfants ayant des besoins particuliers soient l'objet de manifestations d'intolérance de la part des élèves des classes ordinaires. C'est pourquoi, en 1976, le ministère de l'Éducation du Québec met en place une organisation des services selon un système en cascade, depuis les classes spéciales à l'inclusion scolaire en passant par une approche d'intégration. Environ $90 \%$ des élèves sourds sont inclus dans les classes ordinaires ${ }^{15}$, où l'enseignement est médiatisé par un interprète, mais de manière orale, en articulant sans voix ou en signes, plutôt qu'en langue des signes. Il n'existe pas d'interactions avec des pairs sourds et des modèles adultes sourds ou un enseignant sourd ${ }^{16}$.

$$
+4 \div
$$

6. Charles GaUCHER, Ma culture, c'est les mains. La quête identitaire des Sourds au Québec, Québec, Les Presses de l'Université Laval, 2009.

7. Charles GAUCHER, "Les sourds comme figures de tensions identitaires", Anthropologie et sociétés, vol. 29, n² 2, 2005, p. 151-167.

8. Joanne CRIPPS, "Deaf Culture", http://www.deafculturecentre.ca/Public/Default.aspx?I=294\&n= Deaf+Culture (15 janvier 2013).

9. Robert BINET, LSQ 1, Langue des signes québécoise 1. Notes du cours DCA-1017, Trois-Rivières, Université du Québec à Trois-Rivières, 2000.

10. Ibid.

11. Joanne CRIPPS, "Deaf culture", op. cit.

12. Ibidem.

13. Robert BINET, LSQ 1, Langue des signes québécoise 1..., op. cit.

14. Ministère de l'Éducation du Québec. L'éducation de l'enfance en difficulté d'adaptation et d'apprentissage au Québec. Rapport du Comité provincial de l'enfance exceptionnelle (COPEX), Tome 2, Québec, Gouvernement du Québec, 1976.

15. Diane Veillette, Michael Magner et Annie Saint-PIerre, État de la situation de la langue des signes québécoise, Drummondville, Office des personnes handicapées du Québec, 2005, p. 268.

16. Colette Dubuisson et Rachel Berthiaume, Entre parents, parlons surdité..., op. cit., p. 36. 
Depuis les années 1980, il est admis que les langues signées doivent être considérées comme des langues à part entière ${ }^{17}$. En 1981, la Suède devient le premier pays à reconnaitre la langue des signes de Suède comme première langue d'usage des Sourds suédois ${ }^{18}$. D'autres suivront, tels que le Danemark, la Finlande, la Norvège, l'Ouganda, la France, certains États américains et certaines provinces canadiennes, dont l'Ontario, le Manitoba et l'Alberta, par exemple; mais pas le Québec ${ }^{19}$. À ce jour, en Suède, il est admis qu'il faut distinguer les Sourds et les Sourds oralistes ${ }^{20}$.

Lapproche bilingue voit le jour en même temps que les langues des signes sont reconnues ${ }^{21}$. Elle considère la langue des signes comme langue première et la langue parlée ou écrite comme langue seconde ${ }^{22}$. Par exemple, en Suède, depuis 1983, tous les élèves sourds ont droit à une éducation bilingue et culturelle ${ }^{23}$. L'étude longitudinale de Kerstin Heiling ${ }^{24}$ a permis d'en constater les effets positifs sur la réussite éducative des élèves sourds suédois dans l'acquisition du langage, entre autres sur le plan de l'écriture. Des efforts restent tout de même à faire pour favoriser les compétences de lecture et d'écriture des Sourds par rapport aux entendants et leur inclusion dans les classes ordinaires ${ }^{25}$. Le bilinguisme devient également une nécessité pour nombre de Sourds qui souhaitent fonctionner en société : le français dans le milieu de travail, par exemple, et la langue des signes dans la vie familiale et communautaire ${ }^{26}$. Cependant, le bilinguisme ne semble pas réalisable sans la collaboration des parents ${ }^{27}$.

$$
\div+\div
$$

17. Ibid, p. 81-90.

18. Sangeeta BAGGA-GUPTA, «Explorations in Bilingual Instructional Interaction: A Sociocultural Perspective on Literacy ", Learning and Instruction, vol. 12, n 5, 2002, p. 558.

19. Marguerite BLAIS et Jules DESROSIERS, Quand les Sourds nous font signe. Histoires de sourds, Loretteville, Le Dauphin Blanc, 2003.

20. Monica Werngren-ElgSTRÖM, Ove DEHLin et Susanne IWARSSON, «A Swedish Prevalence Study of Deaf People Using Sign Language: A Prerequisite for Deaf Studies ", Disability and Society, vol. 18, $\mathrm{n}^{\circ} 8,2003$, p. 313 .

21. Daniel DAIGLE, «Faire le point sur les philosophies d'enseignement», Colette DUBUISSON et Daniel DAIGLE (dir.), Lecture, écriture et surdité, Montréal, Les Éditions Logiques, 1998, p. 39.

22. Marguerite BLAIS et Jules DESROSIERS, Quand les Sourds nous font signe..., op. cit.

23. Sangeeta BAGGA-GUPTA, "Aspects of Diversity, Inclusion and Democracy within Education and Research ", Scandinavian Journal of Educational Research, vol. 51, n 1, 2007, p. 11.

24. Kerstin HEILING, Bilingual vs. Oral Education: A Comparison of Academic Achievement Levels in Deaf Eighth-Graders from Two Decades, Communication présentée à l'International Congress on Education of the Deaf, 1995.

25. Sangeeta BAGGA-GUPTA, "Explorations in Bilingual Instructional Interaction... ", op. cit., p. 560.

26. Michel LELIÈVRE et Colette DUBUISSON, "Implanter une approche bilingue/biculturelle», Colette Dubuisson et Daniel DAIGLE (dir.), Lecture, écriture et surdité..., op. cit.

27. Colette Dubuisson et Rachel BerThiaume, Entre parents, parlons surdité..., op. cit., p. 40. 
Au cours des années 1990, le Québec entreprend de réformer le système public par la Loi sur l'instruction publique ${ }^{28}$. Plus particulièrement, la Politique en adaptation scolaire ${ }^{29}$ vise la démocratisation de la réussite scolaire. Dans le même temps, les langues signées sont reconnues dans les institutions universitaires, mais elles restent peu considérées dans les systèmes d'éducation actuels et sont souvent rejetées en tant que médium d'enseignement ${ }^{30}$. Toutefois, en 1998, une expérimentation est mise sur pied au Collège des Sourds et une autre à l'école Gadbois, avec l'ouverture de classes bilingues. L'expérience se répète en 2002 à l'école Esther-Blondin et en 2004 à l'école Lucien-Pagé ${ }^{31}$. Dans le projet expérimental de l'école Gadbois, fondé sur un mode bilingue et biculturel, les résultats en lecture des élèves sourds ressemblent à ceux de la Suède et du Danemark ${ }^{32}$. En effet, en fin de scolarité, les élèves sourds ont des capacités en lecture comparables à celles des entendants ${ }^{33}$.

Actuellement, le système d'éducation bilingue et biculturel de la Suède est unique en ce qu'il rend possible pour toute personne sourde l'accès à la langue des signes, à partir de cinq écoles régionales spécialisées, dès l'âge de un an pour le préscolaire, avec le soutien d'interprètes professionnels ${ }^{34}$. En Norvège, un tiers des élèves sourds sont inclus dans les classes ordinaires avec le soutien d'un interprète en langue des signes, tandis que les autres poursuivent des études dans une école spécialisée ou une classe spéciale ${ }^{35}$. En Australie, les élèves sourds, dans une proportion de $83 \%$, sont inclus dans les classes ordinaires avec un accès limité aux services d'interprétariat, favorisant ainsi un modèle d'éducation oraliste ${ }^{36}$. Les politiques françaises et québécoises n'ont pas non plus contribué à une éducation linguistique et culturelle des enfants sourds, celle-ci étant plutôt perçue comme une entrave à l'inclusion scolaire ${ }^{37}$.

$$
4 \div
$$

28. Gouvernement du Québec, «Loi sur l'instruction publique», http://www2.publicationsduquebec. gouv.qc.ca/dynamicSearch/telecharge.php?type=2\&file=/I_13_3/I13_3.html (15 janvier 2013).

29. Ministère de l'Éducation du Québec, Une école adaptée à tous ses élèves: prendre le virage du succès. Politique de l'adaptation scolaire, Québec, Gouvernement du Québec, 1999.

30. Daniel DAIGLE, "Faire le point sur les philosophies... », op. cit., p. 39.

31. Diane Veillette, Michael Magner et Annie Saint-Pierre, Etat de la situation de la langue des signes québécoise..., op. cit., p. 96-102.

32. Ibid, p. 100.

33. Ibidem.

34. Sangeeta BAGGA-GUPTA, «Explorations in Bilingual Instructional Interaction... », op. cit., p. 558.

35. Merv Hyde, Stein Erik OHNA et Oddvar HJUlSTADT, "Education of the Deaf in Australia and Norway: A Comparative Study of the Interpretations and Applications of Inclusion ", American Annals of the Deaf, vol. $150, \mathrm{n}^{\circ}$ 5, 2005, p. 423.

36. Ibid, p. 419-423.

37. Nathalie LACHANCE et Sophie DALLE-NAZÉBI, «La reproduction d'un groupe culturel extra-familial. Territoire et reconstruction de réseaux de transmission entre Sourds ", Diversité urbaine, vol. 7, $\mathrm{n}^{\circ} 2$, 2007, p. 9. 
S'ouvre ainsi la possibilité (si toutefois les moyens sont au rendezvous $^{38}$ ) d'une politique de droit ${ }^{39}$ proposée à l'élève sourd et à sa famille, qui favoriserait un véritable choix dans l'orientation scolaire et professionnelle. La volonté est de permettre une prise de décision et de participation, c'està-dire un empowerment ${ }^{40}$. Une telle perspective suppose de ne pas réduire la notion de "handicap " à la seule déficience ou aux incapacités ${ }^{41}$, mais plutôt de tenir compte de la diversité des situations que les personnes sourdes peuvent vivre. En ce sens, les concepts en jeu dans le "processus de production du handicap ${ }^{42}$ » peuvent permettre d'aborder la réalité tout en reconnaissant la spécificité culturelle et linguistique des personnes sourdes.

Dans une société où la culture ne se transmet plus seulement par l'oral, la littératie, ou «la maitrise de la lecture et de l'écriture et des usages sociaux de l'écrit ${ }^{43}$ ", joue un rôle prépondérant pour favoriser la participation sociale des personnes sourdes dans les domaines de l'éducation et du travail. Le concept de participation sociale ${ }^{44}$ permet d'aborder le "handicap" comme un fait social, non lié exclusivement aux seuls systèmes organiques: c'est un phénomène situationnel. Le concept de participation sociale se définit en termes de degré de difficulté, de type d'aide requis et de niveau de satisfaction à la réalisation des activités courantes ou des rôles sociaux valorisés, tels que l'éducation ou le travail, par exemple. La participation sociale de la personne sourde est soumise à des obstacles ou à des facilitateurs présents dans son environnement.

Des obstacles dans la famille, à l'école et dans la communauté peuvent entraver le développement de la littératie chez l'élève sourd et donc sa participation sociale. Plusieurs pratiques parentales peuvent constituer des obstacles, notamment l'utilisation exclusive de la langue orale et de gestes

$$
4+4
$$

38. Union nationale pour l'insertion sociale du déficient auditif, "Congrès sur la loi du 11 février 2005 et ses enjeux pour les publics de personnes sourdes ou malentendantes et leurs familles ", Echo Magazine, $\mathrm{n}^{\circ} 725,2005$, p. 7.

39. Marie DURIBREUX, «La loi “ handicap ” à l'épreuve", Direction(s), n² 24, 2005, p. 22.

40. Yves VAILlANCOURT, "Les politiques sociales et les personnes ayant des incapacités au Québec", Revue internationale sur les concepts, les définitions et les applications, vol. 13, nos 1 et 2, 2004.

41. Organisation mondiale de la santé, Classification internationale du fonctionnement, du handicap et de la santé. Projet final, 2000.

42. Patrick Fougeyrollas, René Cloutier, Hélène Bergeron, Jacques CÔTÉ et Ginette ST-MiCHEL, Processus de production du handicap. Classification québécoise, Québec, Réseau international sur le Processus de production du handicap, 1998.

43. Sandrine BURGAT, "Un exemple atypique d'acquisition du langage écrit en contexte bilingue: l'enfant sourd locuteur de la langue des signes qui apprend à lire et à écrire en français ", Actes du colloque AcquisiLyon 09, Lyon, 2009, p. 2.

44. Patrick FougeYrollas, René Cloutier, Hélène Bergeron, Jacques Côté et Ginette ST-MiCHEL, Processus de production du handicap..., op. cit. 
limités dans les familles ${ }^{45}$, le manque de soutien à l'apprentissage de la lecture, de l'écriture et de la langue des signes ${ }^{46}$, ou le manque de temps, de connaissances et de compétences pour le développement linguistique de l'enfant sourd à la maison ${ }^{47}$. Les parents sont confrontés à un manque d'information pour l'orientation scolaire de leur enfant sourd, au manque de qualification du personnel scolaire ${ }^{48}$, à des lacunes dans la quantité et la qualité des services d'interprétariat dans les écoles, le milieu de travail ${ }^{49}$.

Des contraintes discriminatoires influent sur le développement linguistique, social, scolaire, professionnel et, par le fait même, sur la situation des personnes sourdes. Les difficultés de lecture et d'écriture peuvent entraver l'intégration au monde du travail et la qualité de vie de la personne sourde ${ }^{50}$. Bon nombre de personnes sourdes sont confrontées à la pauvreté sociale et économique et à la difficulté d'accès à l'éducation et à l'emploi ${ }^{51}$. Un taux élevé d'analphabétisme et une faible scolarisation affectent encore la communauté sourde au Québec ${ }^{52}$. La réussite éducative s'avère difficile pour environ la moitié des personnes sourdes. En effet, $45 \%$ d'entre elles au Québec ne possèdent aucun diplôme - deux fois plus que chez les entendants - et se retrouvent inactives ou au chômage ${ }^{53}$.

En somme, il existe un certain nombre d'obstacles dans l'environnement des personnes sourdes qui construisent un problème spécifique

$$
\stackrel{+\div}{+\div}
$$

45. Katrina R. MILlER, Forensic Issues of Deaf Offenders, Beaumont, Lamar University, 2001, p. 61.

46. Susan FOSTER et Janet MACLEOD, "The Role of Mentoring Relationships in the Career Development of Successful Deaf Persons ", Journal of Deaf Studies and Deaf Education, vol. 9, n 4, 2004, p. 449.

47. Alice ERIKS-Brophy, Andrée DurieuX-Smith, Janet OLDS, Elizabeth FitZPATRICK, Cheryll DUQUETTE et Joanne WHITTINGHAM, "Facilitators and Barriers to the Inclusion of Orally Educated Children and Youth with Hearing Loss in Schools : Promoting Partnerships to Support Inclusion ", Volta Review, vol. 106, n 1, 2006, p. 70.

48. Vicki L. WOLFE, An Investigative Study Identifying the Factors which Influence Parents as They Make Educational Placement Decisions for their Children who Are Deaf, Tennessee, The University of Tennessee, 1999, p. 85-91.

49. Plusieurs auteurs appuient la présence d'un tel contexte au Québec, dont Marguerite Blais (Variantes sur la culture sourde..., op. cit., p. 35-36), le Centre québécois de la déficience auditive (Avis du CQDA sur l'organisation et la gestion des services d'interprétation visuelle, Montréal, CQDA, 2009, p. 8-9), Dominique Pinsonneault et Martin Bergevin (Enquête sur la formation et l'emploi en déficience auditive au Québec. Version intégrale, Montréal, Centre québécois de la déficience auditive, 2006, pp. 83 et 161), ainsi que Diane Veillette, Michael Magner et Annie Saint-Pierre (État de la situation de la langue des signes québécoise..., op. cit., p. 216-237).

50. Daphné A. DuCHARme, Isabelle ARCAND et Julie CHRÉTIEn, "Le Signwriting comme outil pour apprendre le français écrit: une contribution à la construction identitaire de jeunes Sourds en milieu francophone minoritaire?", Reflets: revue d'intervention sociale et communautaire, vol. $16, \mathrm{n}^{\circ} 2,2010$, p. 176.

51. Katrina R. MiLleR, Forensic Issues..., op. cit., p. 14.

52. Marguerite BLAIS, Variantes sur la culture sourde..., op. cit., p. 352.

53. Dominique Pinsonneault et Martin BergeVIn, Enquête sur la formation et l'emploi..., op. cit., p. 43 et 91 . 
pour leur éducation et leur intégration au marché du travail. Quelles sont les perceptions des personnes sourdes, de leurs parents et enseignants à propos des obstacles et des facilitateurs au développement de la littératie et de la participation sociale dans les domaines de l'éducation et du travail ? Il y a lieu de cerner les conséquences que revêt la maîtrise du français, entre autres, au plan de la lecture et de l'écriture, pour des personnes sourdes gestuelles ou bilingues dans des situations reliées à l'éducation ou au travail. Une personne sourde gestuelle utilise de manière privilégiée la langue des signes, alors qu'une personne sourde bilingue s'appuie à la fois sur la langue des signes et la langue orale pour communiquer. Voyons la manière dont les familles, l'école et la communauté facilitent le développement de la participation sociale des personnes sourdes gestuelles ou bilingues au Québec au moyen de la littératie.

\section{INFLUENCE DE L'ENVIRONNEMENT SUR LE DÉVELOPPEMENT DE LA LITTÉRATIE ET DE LA PARTICIPATION SOCIALE DE LA PERSONNE SOURDE}

Le modèle des obstacles et des facilitateurs au développement de la participation sociale ${ }^{54}$ (figure 1) permet de considérer la relation réciproque entre l'individu et l'environnement, avec les obstacles et les facilitateurs, selon un plan rapproché puis plus éloigné, ainsi que le résultat de cette interaction illustrée par la situation de handicap ou de participation sociale. Plus précisément, il est possible de relever des facteurs provenant de la famille, de l'école et de la communauté qui ont une influence sur le développement de la participation sociale de la personne sourde dans les domaines de l'éducation et du travail ${ }^{55}$ et, au fil des années, sur le plan de la littératie.

$$
\div+\div
$$

54. Sylvain LETSCHER, Ghyslain PARENT et Rollande DESLANDES, «Obstacles et facilitateurs à la participation sociale des personnes sourdes dans les domaines de l'éducation et du travail : état de la situation ", Développement humain, handicap et changement social, vol. 18, $\mathrm{n}^{\circ} 2$, 2009, p. 33-34.

55. Dans la continuité de : Sylvain LETSCHER, Ghyslain PARENT et France BEAUMIER, «Un spectacle de danse contemporaine réalisé avec des élèves sourds et des élèves entendants en France: influence du Processus de production du handicap [PPH] de Fougeyrollas et al. (1998) afin de favoriser la participation sociale de personnes sourdes ", Développement humain, handicap et changement social, vol. $17, \mathrm{n}^{\circ} 1,2008$, p. 59-73; Sylvain LETSCHER, Ghyslain PARENT et Rollande DESLANDES, "Obstacles et facilitateurs à la participation sociale... ", op. cit., p. 23-42 ; Sylvain LETSCHER, "Le modèle des obstacles et des facilitateurs au développement de la participation sociale : vers un changement de nos pratiques pour améliorer la participation d'un élève sourd?", http:/www.ripph.qc.ca/userfiles/file/Colloque/Colloque\%202011/ Powerpoint/Conference_6_Sylvain_Letscher.ppt (26 mars 2012); et Sylvain LETSCHER, Ghyslain PARENT et Rollande DESLANDES, "De la résilience à la libération pour l'inclusion d'une personne sourde ", Développement humain, handicap et changement social, vol. 20, ${ }^{\circ} 1,2012$, p. 41-52. 
FIGURE 1.

\section{MODĖLE DES OBSTACLES ET DES FACILITATEURS AU DÉVELOPPEMENT DE LA PARTICIPATION SOCIALE}

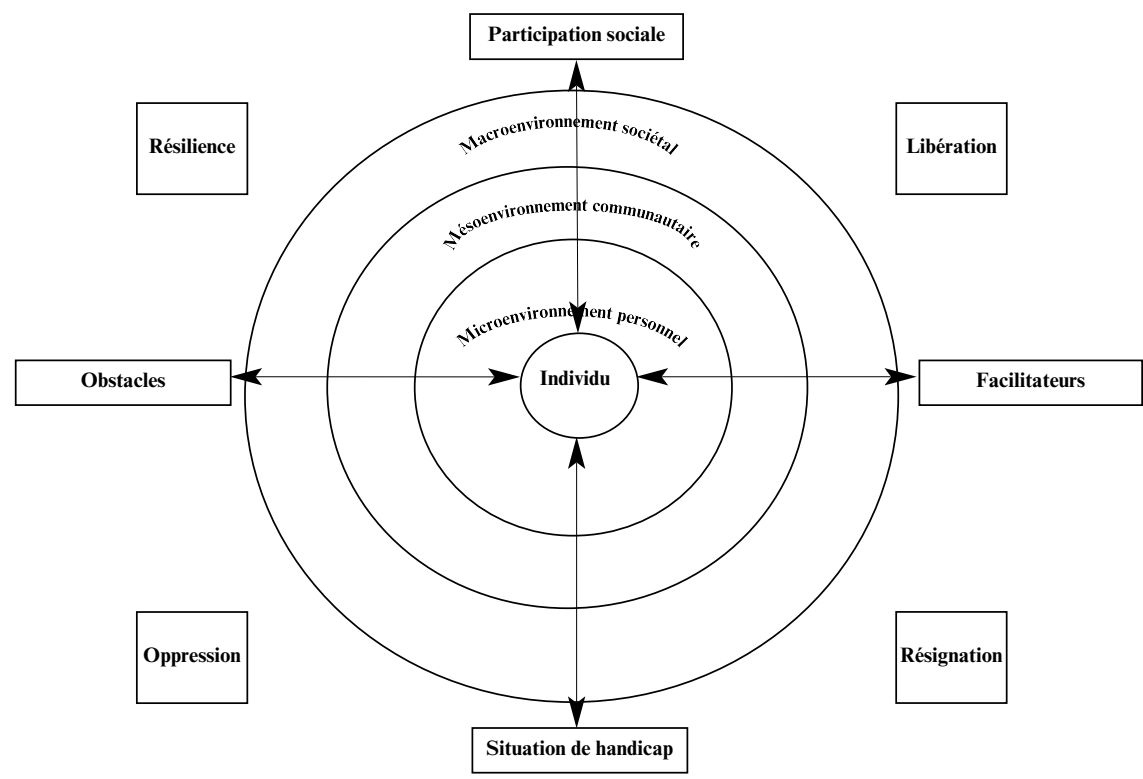

Le macroenvironnement sociétal ${ }^{56}$ comprend les politiques et programmes gouvernementaux. Le gouvernement devrait favoriser, entre autres, un plan d'action qui reconnaît la langue des signes québécoise comme langue d'enseignement et offrir des services appropriés en ce sens ${ }^{57}$. Un organisme ${ }^{58}$ peut aussi avoir un rôle d'information et de sensibilisation au sujet de la surdité, de soutien et de coordination ${ }^{59}$.

$$
+\div
$$

56. Patrick FOUgEYROLLAS, "Comprendre le Processus de production du handicap (PPH) et agir pour la participation sociale, une responsabilité sociale et collective ", http://adep.hautetfort.com/media/00/02/ 593637559.doc (22 mars 2012).

57. Par exemple, Marguerite Blais (Variantes sur la culture sourde..., op. cit., p. 1) ; le Centre québécois de la déficience auditive (Avis du CQDA..., op. cit., p. 8) et Diane Veillette, Michael Magner et Annie SaintPierre (État de la situation de la langue des signes québécoise..., op. cit., p. 382).

58. Le Regroupement des personnes sourdes de la Mauricie, par exemple, assure un tel suivi. Dans le même sens, la Fondation des Sourds du Québec a pu apporter un soutien financier à ce projet de recherche. L'association du Québec pour enfants avec problèmes auditifs (AQEPA) regroupe plus particulièrement des parents ou des intervenants qui visent l'inclusion des jeunes sourds.

59. Dominique Pinsonneault et Martin BergeVIn, Enquête sur la formation et l'emploi..., op. cit., p. 160. 
Le mésoenvironnement communautaire ${ }^{60}$ comporte l'organisation des infrastructures physiques du milieu de vie, de même que, par exemple, les attitudes du personnel scolaire, des collègues ou de l'employeur. Plusieurs facilitateurs à la participation sociale apparaissent, dont l'ouverture du milieu à des personnes sourdes, la proposition de formation à la langue des signes, pour le personnel ${ }^{61}$, et aux stratégies d'enseignement adaptées en matière de lecture et d'écriture pour les enseignants, les programmes individualisés de développement des compétences scolaires, sociales et linguistiques, la sensibilisation des pairs à la surdité ${ }^{62}$ et à la culture sourde ${ }^{63}$, le soutien moral avec des lettres d'encouragement ${ }^{64}$. S'ajoute à cela l'accès aux services d'interprétariat et d'enseignement bilingue ${ }^{65}$.

Le microenvironnement personnel peut comprendre les parents, les proches, les amis, l'enseignant, le conseiller d'orientation, l'employeur ou encore des associés ${ }^{66}$. À titre d'illustration, des "parents défenseurs " apportent du soutien, des conseils, de la flexibilité, des occasions de prendre des décisions et de s'autodéterminer ${ }^{67}$. Des "parents protecteurs " ont un contrôle et une structure de participation élevée, tandis que des parents "absents", ou "distants" ont une faible participation, compensée par un rôle soutenu de l'enseignant par procuration ${ }^{68}$. Les enfants sourds sont alors envoyés dans des écoles spéciales, sur de longues périodes, loin de leur famille, qui dès lors n'assure plus l'éducation du jeune ${ }^{69}$. Le cadre de référence renvoie à la pers-

$$
\div+\div
$$

60. Pamela E. DAVIS-KEAN et Jacquelynne ECCLES, «Influences and Challenges to Better Parent-School Collaborations", Evanthia N. PATRIKAKOU, Roger P. WeISsberg, Sam RedDing et Herbert J. Walberg [dir.], School-Family Partnerships for Children's Success, New York, Teachers College, 2005, p. 62 ; Patrick FougeYrollas, "Comprendre le Processus de production du handicap (PPH)... ", op. cit. 61. Dominique Pinsonneault et Martin Bergevin, Enquête sur la formation et l'emploi..., op. cit., p. 159.

62. Alice ERIKs-Brophy, Andrée DurieuX-Smith, Janet OldS, Elizabeth FitZPATrick, Cheryll DUQUETTE et Joanne WHITTINGHAM, «Facilitators and Barriers to the Inclusion... », op. cit., p. 63-73. 63. Suzette GARAY, "Listening to the Voices of Deaf Students", Teaching Exceptional Children, vol. 35, $\mathrm{n}^{\circ} 4,2003$, p. 46.

64. Brigid M. NoOnan, Susanna M. Gallor, Nancy F. Hensler-McGinnis, Ruth E. Fassinger, Shihwe WANG, Jennifer GoODMAN, "Challenge and Success: A Qualitative Study of the Career Development of Highly Achieving Women With Physical and Sensory Disabilities", Journal of Counseling Psychology, vol. 51, $\mathrm{n}^{\circ} 1,2004$, p. 75.

65. Diane Veillette, Michael MAgner et Annie SAINT-PIERre, État de la situation de la langue des signes québécoise..., p. 383.

66. Pamela E. DAVIS-KEAN et Jacquelynne ECCLES, "Influences and Challenges to Better... », op. cit., p. 62 ; Patrick FougEYROLLAS, "Comprendre le Processus de production du handicap (PPH)... » op. cit.. 67. Lauren Lindstrom, Bonnie DOREN, Jennifer MeTHenY, Pam Johnson et Claire Zane, "Transition to Employment: Role of the Family in Career Development », Exceptional Children, vol. 73, $\mathrm{n}^{\circ} 3,2007$, p. 358-359.

68. Ibidem.

69. Sylvain LETSCHER, Perception de personnes sourdes sur les obstacles et les facilitateurs reliés à la participation sociale dans les domaines de l'éducation et du travail, Montréal, Université du Québec à Montréal, sous presse. 
pective critique de la pédagogie des opprimés ${ }^{70}$. Le processus qui conduit de l'oppression à la libération fait référence à la capacité de la personne à développer une compréhension critique de sa situation, à prendre une décision et à participer à la vie sociale pour que puisse s'instaurer une nouvelle situation vers une pleine participation sociale ${ }^{71}$. Le processus qui conduit de la résignation à la résilience mobilise la capacité de la personne à (re)construire, à (re)créer son identité, qui se développe dans la participation à la culture et dans un engagement en tant quacteur, et non pas seulement en tant qu'assisté $^{72}$. La résistance active $\mathrm{e}^{73}$ est un processus qui conduit de l'oppression et la résignation à la résilience et la libération. La résistance active peut donc se définir comme la capacité de la personne à avoir une compréhension critique de sa situation, à construire son identité, à s’engager dans la société et, par là, à influencer le développement de sa participation sociale.

Le modèle des obstacles et des facilitateurs au développement de la participation sociale ${ }^{74}$ peut être exprimé sous la forme d'une représentation graphique $^{75}$ qui situe la personne dans l'un ou l'autre des quadrants. Dans le quadrant 1, une personne peut être dans un environnement avec beaucoup d'obstacles, mais avoir une forte participation sociale du fait de sa résilience, par exemple lorsqu'elle n'obtient aucun soutien de sa famille mais réussit tout de même à se réaliser et à s'épanouir dans la société. La présence de facilitateurs dans l'environnement de la personne permet une augmentation de sa participation sociale comme le présente le quadrant 2. Cela peut être le cas lorsque le gouvernement rend accessibles des services pour faciliter la poursuite des études. À l'inverse, dans le quadrant 3, la présence d'obstacles et leur influence sur la personne entravent sa participation sociale. Par exemple, des attitudes discriminantes peuvent l'empêcher de s'inscrire pleinement sur le marché du travail. Le quadrant 4 permet de remarquer que, malgré la mise en place de facilitateurs dans l'environnement de la personne, celle-ci peut tout de même être en situation de handicap. Par exemple, il est proposé à la personne de s'engager dans un organisme de défense des droits, mais elle refuse.

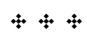

70. Paulo FreIRE, Pédagogie des opprimés: suivi de conscientisation et révolution, Paris, François Maspero, 1974.

71. Ibidem, et Yves VAILLANCOURT, "Les politiques sociales et les personnes ayant des incapacités... ", op. cit.

72. Boris CYRULNIK, Les vilains petits canards, Paris, O. Jacob, 2001.

73. Ibidem.

74. Sylvain LETSCHER, Ghyslain PARENT et Rollande DESLANDES, «Obstacles et facilitateurs à la participation sociale...", op. cit., p. 33-34.

75. Sylvain LETSCHER, Ghyslain PARENT et Rollande DESLANDES, «De la résilience à la libération... ", op. cit., p. 41-52. 


\section{MÉTHODOLOGIE DE RECHERCHE}

La méthodologie consiste à privilégier l'étude qualitative de divers cas. Au total, 22 répondants ont participé à l'étude, dont sept personnes sourdes, six mères, deux pères et sept enseignants. La triangulation est la méthode de validation la plus efficace pour l'étude de $\operatorname{cas}^{76}$. Les participants sourds ont rempli un questionnaire sur la «mesure des habitudes de vie $" 77$ dans les domaines de l'éducation (14 énoncés) et du travail (12 énoncés) et sur la "mesure de la qualité de l'environnement ${ }^{78}$ dans les domaines du soutien et des attitudes de l'entourage, du marché du travail et des services éducatifs (26 énoncés). Le questionnaire permet de mieux cerner le profil de participation sociale des participants dans les domaines de l'éducation et du travail, reliés aux obstacles et aux facilitateurs présents dans l'environnement de la personne. Le questionnaire a pu servir de grille pour l'analyse des entrevues effectuées auprès des participants sourds. Un questionnaire sur les facteurs de soutien parental relatifs au choix de carrière ${ }^{79}$ (27 énoncés) a également été soumis aux parents des participants. Un récit de vie de type narratif sous forme d'entrevue individuelle structurée ${ }^{80}$ a été réalisé à partir du mois d'octobre 2009 dans le local d'une association pour les participants sourds, avec le soutien d'un interprète professionnel. Il faut souligner que l'interprète professionnel s'identifie aux CODAs (children of deaf adults), c'est-à-dire en tant que personne issue de parents qui sont sourds et ayant une identité bilingue et biculturelle, sur la base de la langue des signes et la langue orale $^{81}$. Les parents et les enseignants ont été joints soit à domicile, soit dans l'école du milieu. Le questionnaire et le canevas d'entrevue remis aux participants sourds ont été traduits en français sourd par le Regroupement des personnes sourdes de la Mauricie. La méthode d'analyse de contenu est

$$
\div+\div
$$

76. Thierry KARSENTI et Stéphanie DEMERS, «L'étude de cas », Thierry KARSENTI et Lorraine SAVOIEZAJC (dir.), La recherche en éducation: étapes et approches, Sherbrooke, Les Éditions du CRP, 2004, p. 236; et Sharan B. MERriam, Qualitative Research and Case Study Applications in Education, San Francisco, Jossey-Bass, 1998, p. 40.

77. Patrick FoUgeyrolLAS et Luc NOREAU, Mesure des habitudes de vie (MHAVIE 3.0), Québec, Réseau international sur le Processus de production du handicap, 1998, p. 1-30.

78. Patrick Fougeyrollas, Luc Noreau, Ginette Saint-Michel et Kathryn Boschen, Mesure de la qualité de l'environnement (Version 2.0), Québec, Réseau international sur le Processus de production du handicap, 1999, p. 1-20.

79. Sherri L. TURnER, Annette Alliman-Brissett, Richard T. LAPAN, Sharanya UdiPI et Damla ERgun, "The Career-Related Parent Support Scale», Measurement and Evaluation in Counseling and Development, vol. 36, 2003, p. 83-94.

80. Une version adaptée de : Lauren LINDSTROM, Bonnie DOREN, Jennifer METHENY, Pam JOHNSON et Claire ZANE, "GAPS Data Collection Materials », University of Oregon, 2009, p. 1-13.

81. Thomas H. BuLL, Les problèmes de la famille sourde: les CODA et l'identité. Communication présentée à la Première conférence canadienne sur la santé mentale et la surdité, Ottawa, 2004. 
employée par codage et catégorisation, les propos des répondants étant codés en unités de sens. Le codage a été effectué avec l'assistance du logiciel QSR N’Vivo 8.

\section{SYNTHĒSE DES RÉSULTATS}

La synthèse des résultats met d'abord en évidence des caractéristiques personnelles des participants sourds. Ces caractéristiques sont le résultat d'une influence des obstacles et des facilitateurs dans l'environnement, du niveau de participation sociale (ici, dans les domaines de l'éducation et du travail) ainsi que du développement de la littératie de la personne sourde. Les caractéristiques personnelles se rapportent au plan sociodémographique, scolaire, professionnel et relationnel, en termes d'oppression, de résignation, de résilience ou de libération ${ }^{82}$. Cette étude s'appuie sur l'analyse de sept cas, qui concernent cinq hommes (Amélien, Gabriel, Maxens, Léonard, Jacques) et deux femmes (Amandine, Catherine) ${ }^{83}$. Trois participants ont respectivement 22, 32 et 35 ans (Amandine, Maxens et Amélien), quatre ont 38, 40, 42 et 52 (Gabriel, Catherine, Jacques et Léonard). La tranche d'âge de 21 à 35 ans fait partie de la génération d'élèves sourds qui a pu bénéficier des premiers services d'interprétariat dans les écoles (en région) qui ont été mis en place dans les années 1980. Quatre répondants utilisent la langue des signes québécoise (LSQ) et la langue orale (Amélien, Léonard, Catherine, Jacques), alors que les trois autres utilisent la première de manière privilégiée (Amandine, Gabriel, Maxens). Plusieurs participants s'identifient à la fois à la culture sourde et à la culture entendante (Amandine, Amélien, Maxens), les autres s'identifient plutôt à la culture sourde (Gabriel, Léonard, Catherine, Jacques).

Aucun participant n'a fait d'études postsecondaires, bien qu'Amandine ait tenté d'obtenir un diplôme d'études professionnelles. Seulement trois participants ont obtenu un diplôme dans un parcours ordinaire (Amandine) ou de formation professionnelle (Léonard, Jacques). Les autres ont suivi un parcours en adaptation scolaire sans pouvoir obtenir un diplôme (Amélien, Gabriel, Maxens, Catherine). Deux participants ont poursuivi des études dans l'institution spécialisée d'un grand centre (Léonard, Catherine). Amandine est la seule, parmi les participants sourds, à avoir poursuivi des études dans un contexte d'inclusion scolaire, avec un interprète en LSQ, à

$$
\div \div \div
$$

82. Sylvain LETSCHER. Perception de personnes sourdes..., op. cit.

83. Les prénoms des participants sont fictifs. 
partir de sa quatrième année du primaire, dans le milieu ordinaire avec des élèves sourds et des élèves entendants, puis en étant la seule Sourde. Quatre participants ont un emploi (Amandine, Amélien, Léonard, Jacques), alors que trois autres sont soit sans emploi (Gabriel, Catherine), soit au chômage (Maxens). Trois participants sont employés dans le secteur de l'entretien ménager (Amandine, Amélien et Léonard) tandis qu'un autre, Jacques, travaille comme ébéniste.

Les caractéristiques scolaires mettent d'abord en jeu des facteurs propres au profil de résignation, d'oppression ou encore de résilience des participants sourds. Tous, sauf Amélien, évoquent avoir été confrontés à la difficulté d'apprentissage du français, que ce soit de manière orale ou écrite: "Lorsque j'ai été intégrée, cela allait super bien, sauf en français. J'avais de la difficulté à comprendre" (Amandine), "C'était le cas presque pour chaque mot, il fallait faire beaucoup d'efforts pour en trouver le sens [...]. En français, je ne suis pas trop bon" (Jacques). Tous les participants sourds mentionnent la difficulté d'accès à des services d'interprétariat en LSQ au primaire ou au secondaire. Le père d'Amandine met en cause le contexte d'organisation des services, à partir de la commission scolaire, marqué par la difficulté à reconnaître la LSQ comme première langue d'apprentissage : «Au départ, la commission scolaire n'était pas équipée ici [...] La LSQ n'était pas reconnue [...] Écoute, la qualité d'interprétariat est à redire. À l'école primaire, Amandine connaissait plus de signes que ses professeurs, que ses interprètes des fois ». Selon l'enseignant de Catherine, les élèves sourds rencontrent des difficultés d'apprentissage sur la base du français comme première langue. L'utilisation de la LSQ devient un prérequis pour le développement global du langage, le premier média même pour l'apprentissage du français, selon l'enseignante de Maxens.

D'après l'enseignant entendant d'Amandine, l'interprète est la personne la plus importante pour la progression de l'élève sourd dans le milieu ordinaire. Il souligne qu'il est important que l'enseignant entendant utilise les services de l'interprète afin de mieux comprendre la culture sourde, de traduire en signes ses paroles et même d'apprendre des mots en signes, d'adapter son enseignement et ses modalités d'évaluations. Par exemple: "C'est sûr qu' [Amandine] écrivait un peu dans sa langue. Elle faisait le travail et l'interprète corrigeait ensuite pour me dire que telle chose, cela veut dire ça. C'est [l'interprète] qui corrigeait et me donnait la note. [L'interprète] faisait la correction ». L'orthopédagogue-interprète facilite aussi l'accès à l'information pour la personne sourde en transcrivant du vocabulaire spécifique en signes pour transmettre de l'information complexe (Léonard). Selon le père 
d'Amandine, il existe un manque d'adaptation de l'enseignement bilingue et biculturel auprès de l'élève sourd. Cette adaptation doit favoriser l'interaction de l'élève avec des pairs sourds du même âge et avec un modèle adulte sourd ayant un rôle linguistique et pédagogique, ou un enseignant sourd, à l'école. Dans un contexte inclusif, l'orthopédagogue interprète qui a un accès privilégié à la fois à la culture sourde et la culture entendante peut jouer un rôle de médiateur linguistique et pédagogique. Par exemple, il est possible de faire référence à des interprètes entendants qui se reconnaissent comme CODAs (children of deaf adults). Il est aussi possible de faire appel à des interprètes sourds qui ont accès à la communication orale et gestuelle en LSQ. Des Sourds peuvent également être des personnes ressources, des enseignants même, et permettre l'approfondissement de la langue des signes québécoise dans le milieu scolaire. Les interprètes entendants doivent avoir atteint un niveau 2 à 3 d'interprétation gestuelle et se munir d'une formation d'appoint en psychopédagogie. D'après Jacques, le gouvernement doit faciliter une approche d'enseignement bilingue et biculturel. Dans l'école où s'est déroulée le projet "S'entendre par la danse contemporaine ${ }^{84}$ », les enseignants, le personnel scolaire entendant, les élèves entendants et les élèves sourds ont pu avoir accès à une formation en langue des signes avec un enseignant sourd. Un médiateur linguistique et pédagogique sourd, ayant accès à la langue orale et gestuelle, soutient les élèves sourds dans la classe ordinaire. Les élèves sourds ont même fait une présentation par affiche dans l'école sur la culture sourde, l'alphabet en signes et les arts par les Sourds.

L'inadéquation du plan de services $^{85}$ dans l'adaptation de l'approche de communication peut rendre difficile la compréhension des structures de la langue, tant du français que de la LSQ, d'après l'interprète de Gabriel. Gabriel n'a pu s'approprier une langue, dès le primaire, en raison de l'utilisation simultanée de plusieurs modes de communication, autres que la langue des signes québécoise (LSQ) : «C'était au primaire [...]. Au niveau des signes [...], nous utilisions la LSF (Langue des signes française), l'ASL (American sign language), la communication totale, le français signé». La langue des signes est efficace pour faire comprendre l'information au jeune,

$$
\div \div
$$

84. Sylvain LETSCHER, Ghyslain PARENT et France BEAUMIER, "Un spectacle de danse contemporaine réalisé avec des élèves sourds et des élèves entendants en France : influence du Processus de production du handicap [PPH] de Fougeyrollas et al. (1998) afin de favoriser la participation sociale de personnes sourdes ", Développement humain, handicap et changement social, vol. 17, $\mathrm{n}^{\circ} 1$, 2008, p. 59-73.

85. Le plan de services précise les objectifs, les interventions et les ressources nécessaires entre le réseau de l'éducation et celui de la santé et des services sociaux (Ministère de l'éducation, du loisir et du sport, «Le plan de services individualisé et intersectoriel ", Québec, Gouvernement du Québec, 2006, p. 6). 
permettre la communication avec d'autres Sourds et favoriser l'ouverture, plutôt que le repli sur soi, par l'intégration dans la communauté sourde, selon un enseignant proche de Gabriel. Un tel contexte a pu avoir des conséquences négatives sur la qualité des services d'interprétariat et ainsi provoquer une perturbation grave de la participation sociale de tous les participants, sauf dans le cas d'Amélien, ne serait-ce que pour suivre un cours dans une classe et, pour Catherine, Maxens, Gabriel et Léonard, pour faire des examens: "Comme il n'y avait pas d'interprète, c'était plus difficile d'apprendre le français» (Maxens), "Avec l'aide de l'interprète, cela allait, mais lorsque je me retrouvais seule à l'examen, j'avais de la difficulté à comprendre le sens des mots» (Catherine).

La difficulté d'accès au français écrit ou oral a pu jouer un rôle dans la décision que tous les participants ont prise d'arrêter, d'interrompre ou de ne pas poursuivre leurs études. Il faut remarquer le manque de confiance en soi, l'incertitude dans l'orientation scolaire, la crainte par rapport à la communication dans le choix d'orientation d'Amandine. Ou encore le renfermement de Maxens sur lui-même, puis un sentiment d'isolement et d'exclusion qu'il partage avec Léonard lors de son inclusion sans services d'interprétariat, par exemple. La difficulté de communication peut rendre difficile l'inclusion dans le milieu ordinaire, d'après Léonard. Catherine fait part de son sentiment d'échec, engendré selon elle par l'apprentissage tardif des signes, à 13 ans, lors de son transfert en institution spécialisée, plutôt qu'en cursus ordinaire. D'après l'enseignant de Léonard, l'apprentissage tardif de la langue des signes, débutant au primaire et se poursuivant au secondaire, risque de réduire l'accès à la lecture et à l'écriture, des compétences nécessaires pour l'accès aux connaissances et pour l'inclusion dans le milieu. Pour plusieurs participants (Amélien, Catherine, Jacques), l'organisme de défense des droits de la personne sourde a pu permettre de commencer l'apprentissage de la LSQ à l'âge adulte au moyen d'une formation avec un interprète et des activités de regroupement avec des Sourds.

Plusieurs participants sourds considèrent le soutien de la famille comme un facilitateur mineur (Jacques), moyen (Maxens) et majeur (Amandine). Pour Amélien, dont la conjointe est entendante, la situation familiale joue un rôle de facilitateur majeur. Les parents des participants mettent en jeu un style parental «défenseur» (Amandine, Amélien, Jacques), "protecteur " (Gabriel) ou "distant» (Maxens, Léonard, Catherine). Les parents ont pu faciliter l'accès aux services d'éducation gestuelle et orale (Amandine, Amélien, Gabriel, Catherine, Jacques), aux services d'interprétariat et à l'adaptation du matériel pédagogique (Amandine), à de l'information auprès 
d'associations de parents, des associations de Sourds (Jacques) et pour aider à mieux comprendre la surdité et la culture sourde (Gabriel). L'aide aux devoirs et leçons prend également de l'importance, d'après Amandine et Gabriel, dans l'apprentissage de la lecture, de l'écriture, des signes et de l'oralisme, par exemple. Dans le cas d'Amandine et de Gabriel, les parents ont favorisé l'accès à la langue des signes, à des Sourds adultes et à des entendants.

Tous les participants sourds retiennent des facteurs de libération propres aux caractéristiques scolaires. Tous, sauf Jacques, évoquent l'importance d'obtenir un diplôme du secondaire dans le cheminement ordinaire ou en éducation aux adultes, voire de poursuivre des études postsecondaires jusqu’au cégep ou à l'université, comme le présente Amandine :

Je suis contente d'avoir eu mon secondaire 5, parce que j'ai travaillé fort à l'école au niveau des études, des devoirs [...] Je suis fière parce que j'ai eu mon diplôme [...] Maintenant, je comprends l'importance d'aller à l'école $[\ldots]$ D'aller $[\ldots]$ à l'Université, $[\ldots]$ au cégep ou de faire un DEP [...] Avant, je ne trouvais pas ça important. Je trouvais ça inutile. Actuellement, lorsque je vois les personnes qui ont un bon emploi, j'ai compris que c'est important d'y aller.

Jacques met en évidence le besoin d'interaction, de communication avec les Sourds, voire l'importance de la culture sourde, de l'apprentissage de la langue des signes à un niveau avancé : «Si nous avions eu une formation plus spécifique au niveau de la culture sourde [...] avec un groupe de Sourds [...], cela aurait peut-être été possible d'aller plus loin [...] Il aurait fallu que ce soit de la LSQ avancée». En même temps, l'inclusion dans le milieu ordinaire, l'ouverture, la communication, l'apprentissage du français avec des élèves entendants sont nécessaires selon Maxens et Léonard : «J'aurais aimé ça être plus intégré, mais je ne pouvais pas [...] suivre les mêmes cours que le régulier» (Maxens), "J'ai préféré être avec des élèves entendants pour apprendre à nous connaître. Être dans un ghetto sourd, je trouvais cela ennuyant" (Léonard). Les amis d'Amandine ont pu la soutenir en échangeant avec elle, ce qui lui a permis de développer son apprentissage du français à partir de messages textes MSN. Elle a pu entretenir une relation proche avec des amis entendants. Sa meilleure amie se destine du reste à devenir interprète. Le cheminement peut être propice à l'intégration de l'identité sourde; et la culture entendante, au développement de la personne, comme le souligne Amélien : "J'utilise la langue des signes et le français [...] J’ai intégré les deux, les Sourds, les entendants» (Amélien). 
Gabriel souligne le besoin d'aller chercher de l'aide en lecture et en écriture. La direction d'établissement peut devenir proactive en favorisant un aménagement des cours afin de faciliter l'apprentissage du français, d'après le père d'Amandine: «On a réussi à couper dans ses cours d'anglais [...], à faire transformer des cours grâce au directeur qui n'était pas gêné de passer à côté des portes, par exemple, en changeant le cours de religion en cours de français ». L'enseignante de Maxens met en évidence l'importance de l'orthopédagogue pour développer l'écriture, une compétence nécessaire à l'inclusion sur le marché du travail, par exemple pour faire des $\mathrm{CV}$, des lettres de présentation, pour écrire des notes et des bons de commande. Selon les dires de l'enseignant de Jacques, l'orthophoniste peut aider à développer, dans certains cas, des compétences de communication pour faciliter l'inclusion de l'élève sourd sur le marché du travail.

Les participants sourds mettent à l'épreuve leur résilience non seulement sur le plan scolaire, mais aussi sur le plan professionnel, ce qui se traduit là aussi sous forme d'oppression ou de résignation. Tous les participants, sauf Amélien et Maxens, évoquent avoir vécu des difficultés dans la communication avec les personnes de leur milieu de travail. Amandine et Jacques font part d'un sentiment d'isolement, de marginalisation. Gabriel mentionne une baisse de confiance en soi, la peur de se faire blâmer. La communication est difficile dès l'entretien d'embauche, comme le précise Catherine. La recherche d'un emploi régulier a pu occasionner une perturbation grave pour Amandine, Gabriel, Maxens et Catherine. Plus encore, Amandine, Jacques et Maxens font part de frustrations, de conflits ou encore de discrimination salariale. Le manque de soutien de l'employeur peut être une source de discrimination, comme l'illustre bien l'exemple de Jacques:

Lors de mon ancien emploi, j'ai demandé au chef d'entreprise d'engager un interprète lors des réunions $[. .$.$] en lui donnant une lettre$ d'information, que je pouvais obtenir des services d'interprétariat auprès du [Service d'interprétariat régional] [...] Il ne l'a jamais fait [...] Tout le monde pouvait comprendre ce qu'il disait, sauf moi [...] Je suis resté un peu, puis dès qu'un nouvel employé arrivait, il avait un salaire plus élevé que le mien, malgré l'expérience qu'il avait. Le patron ne faisait rien pour moi [...] J'étais pas mal frustré [...] J'étais déçu. J'ai décidé de travailler ailleurs. Cela allait bien, mais je n'étais pas d'accord avec le niveau salarial [...] Si j'avais su avant comment procéder, j'aurais probablement fait des plaintes pour discrimination.

Plusieurs obstacles apparaissent dans la relation avec l'employeur chez tous les participants, sauf Amélien. L'employeur est susceptible de manifester, 
entre autres, de la peur et de la difficulté à communiquer avec un Sourd, un manque de sensibilisation à la culture sourde, ou bien de la réticence à engager un interprète lors des réunions pour faciliter la transmission de l'information. D'après le père d'Amandine, sa fille peut avoir à l'occasion un interprète quand il y a des nouveautés dans la tâche, des réunions syndicales, par exemple, pour avoir une bonne compréhension de son milieu. Cependant, le père et la mère d'Amandine soulignent le manque de services d'interprétariat au service externe d'aide à la main d'œuvre, rappelant en même temps la pénurie d'interprètes à la pige. Jacques souligne l'absence de sensibilisation du milieu de travail. À l'instar de Gabriel et de Catherine, il relate sa situation de handicap complet à interagir avec ses collègues, tandis que Maxens a une perturbation grave et Amandine, une perturbation modérée.

Les participants sourds relatent néanmoins de facteurs de libération sur le plan professionnel. À l'exception de Maxens et de Catherine, ils ont pu avoir des expériences positives au cours de leur emploi, comme dans la communication avec les personnes du milieu d'emploi, qui peut être améliorée par la présence d'un collègue sourd. Par exemple, Amandine raconte: "À [l'entreprise], mon ami est Sourd. Cela a été super facile au niveau de la communication [...] Une employée me montrait avec des gestes, c'était visuel aussi et puis moi je suis très visuelle». La sensibilisation du milieu de travail d'Amandine, sans doute lié à la présence de sa sœur cadette dans l'entreprise, lui a permis de valoriser ses compétences, son mode de communication et son autonomie. Le soutien des collègues s'est exprimé lorsqu'ils communiquaient un peu par signes avec elle ou lui référaient un client sourd.

Les expériences positives que vivent les participants sourds sur le marché du travail peuvent favoriser le développement de la confiance en soi, l'autonomie, la prise de responsabilité et l'estime de soi, la capacité à défendre ses droits et à faire du mentorat: "[Une collègue entendante] m'a dit que c'était facile avec moi, que j'étais capable de comprendre. Je suis capable de faire ça toute seule» (Amandine), "J'ai réussi avec [une personne sourde]. Elle voulait quitter l'école. Je l'ai convaincue de continuer l'école et c'est grâce à moi qu'elle a eu son diplôme en secondaire 5 . Si je ne le lui avais pas conseillé, elle aurait décroché de l'école. Je l'ai poussée. Je l'ai encouragée" (Amélien). Gabriel a pu dépasser les problèmes de communication avec son employeur grâce à son expertise : "J'ai travaillé [...] dans un garage automobile [...] J'étais capable de me débrouiller avec le patron. Lorsqu'il me demandait de changer telle ou telle pièce, cela allait bien. Je ne faisais pas d'erreur». L'exemple de Jacques montre que le fait de travailler pour un employeur sensibilisé à la culture sourde permet d'outrepasser les préjugés et 
les difficultés liées à la communication et de se démarquer par rapport aux collègues entendants :

Lorsque je ne comprends pas quelque chose, je demande à mon patron, par exemple quand on de la difficulté à communiquer ensemble avec les employés. Je demande à mon patron de m'aider, parce qu'il connaît un petit peu les Sourds. Je communique plus avec lui [...] Certains communiquent avec moi par écrit pour que cela soit plus facile pour moi [...] Lorsque l'on a changé de machine, le patron m'a montré la base [...] J'avais la curiosité d'apprendre comment cela fonctionnait [...] Moi, je suis plus visuel [...] Cela n'a pas été facile, mais j’ai appris. Les autres sont restés surpris que je sois le seul à savoir l'utiliser [...] Il n'y a que le patron et moi qui savons comment utiliser la machine $[\ldots]$ Je suis satisfait.

L'employeur peut faciliter la participation de la personne sourde dans le monde du travail. Cela commence bien sûr par la volonté manifestée d'embaucher une personne sourde. L'enseignant de Catherine affirme que le lien étroit qui peut s'établir entre l'école et les employeurs facilite le soutien auprès du jeune. D'après tous les participants, le fait que les employeurs démontrent une attitude positive constitue des facilitateurs. Amandine a pu obtenir des services d'interprétariat par l'intermédiaire de son employeur dès le premier mois de son embauche, lors de son premier emploi. L'employeur peut faire appel au Service régional d'interprétariat pour obtenir un interprète à des moments clés, tels que l'embauche, ou des réunions, comme le mentionne Amélien. Cette démarche peut être soutenue par le Service externe d'aide à la main d'œuvre (SEMO) qui sensibilise le personnel entendant du milieu de travail au sujet de la surdité, de la culture sourde et des stratégies de communication adaptées. L'employeur peut se montrer intéressé à suivre une courte formation en langue des signes québécoise. De tels efforts facilitent la communication orale et gestuelle avec la personne sourde, selon Léonard.

\section{DISCUSSION}

L'analyse qualitative a permis de décrire des obstacles et des facilitateurs au développement de la littératie et de la participation sociale de la personne sourde dans les domaines de l'éducation et du travail. La perspective de collaboration école-famille-communauté renvoie à l'influence du macroenvironnement sociétal, du mésoenvironnement communautaire et du microenvironnement personnel sur le développement de la participation 
sociale ${ }^{86}$, de même que les caractéristiques propres à la littératie chez la personne sourde. Les résultats permettent de mettre en évidence la relation de la personne sourde avec plusieurs acteurs clés, qui conduisent soit à un profil de résignation et d'oppression, soit à un profil de résilience ou de libération.

Les participants illustrent les quatre profils d'influence de l'environnement sur la participation sociale ${ }^{87}$ selon: le quadrant 1 , "Résilience ", avec Léonard et Jacques; le quadrant 2, «Libération», avec Amandine et Amélien; le quadrant 3, "Oppression", avec Gabriel et Catherine; et le quadrant 4, "Résignation», avec Maxens. Dans cinq cas (Amandine, Gabriel, Maxens, Catherine, Jacques), il faut relativiser la nature des profils. Le processus est plus dynamique que statique. Ainsi, Amandine et Jacques vivent à la fois de la résilience et de la libération. Maxens est sujet à de l'oppression et à de la résignation. Une faible résilience apparaît également dans le domaine de l'éducation chez Gabriel et Catherine, malgré leur profil d'oppression. L'absence d'influence positive de l'environnement sur la participation sociale de Léonard empêche une libération. Selon lui, Amélien vit une pleine libération.

Les caractéristiques scolaires et professionnelles sont tributaires des expériences positives et négatives dans le milieu sourd et le milieu entendant. Les résultats rappellent la difficulté que les personnes sourdes rencontrent dans l'apprentissage du français oral et écrit comme première langue, ce qui a pu jouer un rôle décisif dans la décision d'arrêter, d'interrompre ou de ne pas poursuivre les études secondaires. Amandine, une personne sourde gestuelle âgée de 22 ans et qui communique de manière privilégiée avec la langue des signes, est la seule à avoir obtenu un diplôme de cinquième secondaire, puis à avoir poursuivi des études professionnelles. Pourtant, les participants sourds s'accordent sur l'importance qu'il y a à obtenir un diplôme du secondaire dans le cheminement ordinaire ou en éducation aux adultes, voire de poursuivre des études postsecondaires jusqu'au cégep ou à l'université.

Dans le microenvironnement personnel, les styles parentaux de types défenseur, protecteur ou distant et les pratiques parentales, entre autres, peuvent exercer une influence sur le développement de la littératie et de la

$$
+4
$$

86. Tout comme : Sylvain LETSCHER, "Le modèle des obstacles et des facilitateurs... ", op. cit. 87. Les profils sont obtenus à partir des échelles d'appréciation appliquées à la mesure des habitudes de vie et de l'environnement de Patrick Fougeyrollas, Julie Tremblay, Luc Noreau, Serge Dumont et Myreille Saint-Onge ( Les personnes ayant des incapacités : encore plus pauvres et à part... qu'égales. Les facteurs personnels et environnementaux associés à l'appauvrissement des personnes ayant des incapacités et de leur famille», http://www.irdpq.qc.ca/communication/PDF/Encorepluspauvresetapartquegales.pdf (26 mars 2012)) et de la représentation graphique de Sylvain Letscher (Sylvain LETSCHER, "Le modèle des obstacles...", op. cit.). 
participation sociale de la personne sourde. Plusieurs auteurs constatent le besoin de stimulation du langage de l'enfant sourd à la maison dès l'âge préscolaire $^{88}$, notamment en lecture et en écriture ${ }^{89}$. Les parents peuvent apporter du soutien dans l'aide aux devoirs et leçons, comme le montre, par exemple, le parcours de vie d'Audrey Lessard, "la première sourde à être spécialiste de la santé " au Canada ${ }^{90}$.

Le mésoenvironnement communautaire met en évidence, entre autres, des facteurs propres à la direction d'école, aux enseignants, aux interprètes, aux collègues d'études, aux employeurs, aux collègues de travail et aux amis. La direction d'établissement a un rôle déterminant dans la mise en place du plan d'intervention et des services d'interprétariat. L'individualisation du suivi peut faciliter, notamment, le développement des compétences scolaires, sociales et linguistiques du jeune sourd ${ }^{91}$. Les participants sourds mentionnent tous la difficulté d'accès à des services d'interprétariat en $\mathrm{LSQ}^{92}$, alors que la qualité de ce service risque d'influencer la poursuite des études et l'obtention du diplôme ${ }^{93}$. Le plan de transition, s'il est mis en place dès le secondaire ${ }^{94}$ à l'aide d'une personne-ressource, permet de sensibiliser les divers acteurs à la surdité, à la culture sourde et aux accommodements, jusque dans le milieu de travail ${ }^{95}$.

Le rôle de l'enseignant devient significatif lorsqu'il sait qu'il peut s'appuyer sur l'interprète dans la classe ordinaire, avoir recours à une individualisation de l'enseignement et procéder à une adaptation du matériel pédagogique $^{96}$. L'enseignant doit considérer la culture sourde dans les besoins et les intérêts du jeune sourd. Pour obtenir de l'aide, l'enseignant peut se rapprocher des regroupements de personnes sourdes, par exemple ${ }^{97}$. Un ensei-

$$
\div \div \div
$$

88. Alice Eriks-Brophy, Andrée DurieuX-Smith, Janet OldS, Elizabeth FitzPATrick, Cheryll DUQUETTE et JoAnne WHITTINGHAM, "Facilitators and Barriers to the Inclusion... ", op. cit., p. 76.

89. Susan FOSTER et Janet MACLEOD, "The Role of Mentoring Relationships in the Career... ", op. cit., p. 449.

90. Hamel, F. (2009). "Devenir docteure malgré la surdité. Parcours. Une première au Canada", Document remis par le Regroupement des personnes sourdes de la Mauricie, p. 33-35.

91. Alice ERIKS-Brophy, Andrée DurieuX-Smith, Janet OldS, Elizabeth FitZPATRICK, Cheryll DUQUeTTE et JoAnne WHITTINGHAM, «Facilitators and Barriers to the Inclusion... », op. cit., p. 64.

92. À l'instar de Dominique PINSONNEAUlT et Martin BERGEVIn, Enquête sur la formation et l'emploi..., p. 83, p. 161.

93. Centre québécois de la déficience auditive (CQDA), Document de réflexion sur l'état de l'interprétariat visuel et tactile au Québec et la nécessité d'intervenir rapidement, CQDA, 2009, p. 20.

94. Suzette GARAY, "Listening to the Voices... ", op. cit., p. 46.

95. Dominique PINSONNEAUlT et Martin BERGEVIn, Enquête sur la formation et l'emploi..., op. cit., p. 166-167.

96. Susan FOSTER et Janet MACLEOD, "The Role of Mentoring Relationships in the Career ... ", op. cit., p. 449.

97. Suzette GARAY, «Listening to the Voices... », op. cit., p. 46. 
gnant sourd est nécessaire pour l'apprentissage de la LSQ à un niveau avancé. L'orthopédagogue-interprète est un médiateur linguistique et pédagogique dans la classe ordinaire. En orientant l'intervention dans la classe, il devient un enseignant itinérant qui facilite le développement des compétences scolaires, sociales et linguistiques, de même que l'assistance à l'enseignement et à l'interprétariat ${ }^{98}$. L'employeur devient un facilitateur d'abord par son ouverture à engager une personne sourde et, soutenue par un interprète à des moments clés, il sensibilise le milieu à la culture sourde et favorise l'accès à une formation en signes, par exemple. Le contexte doit permettre d'avoir une personne-ressource pour la transmission de l'information, voire l'apprentissage de la langue des signes pour les employeurs et les employés ${ }^{99}$. Des employés, ou un ami sourd ou entendant, peuvent apporter du soutien en ce sens.

L'analyse du macroenvironnement sociétal fait ressortir le parcours varié des participants sourds dès la prise en charge de l'éducation précoce, ce qui aura par la suite une influence sur l'inclusion du jeune au primaire, au secondaire et lors des études postsecondaires. Amandine est la seule à avoir eu accès à la langue des signes à la petite enfance et à avoir poursuivi des études dans un contexte d'inclusion scolaire, à partir de sa quatrième année du primaire, dans le milieu ordinaire avec des élèves sourds. Elle poursuit ses études secondaires avec un interprète en LSQ en étant la seule élève sourde. Puis, elle est la seule, parmi les participants sourds, à avoir poursuivi des études postsecondaires. La plupart des jeunes sourds de 15 à 34 ans, soit environ $76 \%$, poursuivent aujourd'hui des études en milieu ordinaire et sont beaucoup plus nombreux à obtenir un diplôme secondaire, collégial ou universitaire que leurs pairs plus âgés qui ont connu, entre autres, l'institutionnalisation ${ }^{100}$.

Le gouvernement doit investir dans une formation des interprètes qui leur permette d'obtenir les qualifications requises, pour que l'enfant puisse avoir accès à une éducation gestuelle dès la prématernelle, à un service d'accompagnement à domicile, puis à un service continu pour faciliter la participation sociale, plus particulièrement dans les domaines de l'éducation et du travail. L'inclusion du jeune doit permettre le regroupement de spécialistes, dont un enseignant sourd et des pairs sourds, dans une école ordinaire

$$
4+4
$$

98. Alice ERIKS-Brophy, Andrée Durieux-Smith, Janet OldS, Elizabeth FitZPATricK, Cheryll DUQUETTE et Joanne WhITTINGHAM, «Facilitators and Barriers to the Inclusion... », op. cit., p. 64. 99. Dominique Pinsonneault et Martin BergeVIn, Enquête sur la formation et l'emploi..., op. cit., p. 167.

100. Ibid., p. 145. 
du milieu, ou régionale, s'appuyant sur un enseignement bilingue et biculturel $^{101}$, comme cela a pu être le cas, en partie, pour Amandine. Cette perspective s'harmonise mieux à une politique interculturelle, telle qu'elle existe par exemple au Québec ${ }^{102}$ ou en Grèce ${ }^{103}$, avec le cas des élèves gréco-cypriotes. Le multiculturalisme, un système d'intégration privilégié par la politique fédérale, au Canada, retient un mode de diversité culturelle fondé sur l'ethnicité qui renvoie chacun à sa communauté d'origine. Le Québec a plutôt adopté un modèle basé sur l'interculturalisme, qui vise un échange culturel dans le cadre de valeurs communes d'une nation plurielle majoritairement francophone ${ }^{104}$. En Grèce, les élèves apprennent le grec comme une seconde langue et peuvent accéder à des cours en dehors de la classe ordinaire pour faciliter le suivi avec du matériel spécialisé comme des livres, des activités et des exercices spécifiques, et ce, jusqu’à la fin de l'école primaire ${ }^{105}$. Le gouvernement québécois devrait selon nous reconnaître la langue des signes non seulement de manière constitutionnelle, c'est-à-dire comme la langue officielle des Sourds, mais aussi comme première langue d'enseignement. Il faut rappeler que la langue des signes n'est pas encore reconnue au Québec, contrairement à nombre de pays où elle est inscrite comme première langue officielle d'usage des Sourds, par exemple en Suède ${ }^{106}$, au Danemark, en Finlande, en Norvège, en Ouganda et en France ${ }^{107}$. En Ontario, la LSQ et l'American sign language sont mêmes reconnues comme langues d'enseignement ${ }^{108}$. Ainsi, au Québec, seulement la moitié des personnes sourdes considèrent leurs habiletés de lecture (54\% des répondants) et d'écriture $(50 \%$ des répondants) comme bonnes, contrairement à l'Ontario où les habiletés de lectures (78\% des répondants) et d'écriture (65\% des répondants) sont bonnes à excellentes ${ }^{109}$.

$$
4+4
$$

101. À l'instar du projet de recherche-action participatif et collaboratif, conçu et réalisé entre 2004 et 2006, de Sylvain Letscher, Ghyslain Parent et France Beaumier (Sylvain LETSCHER, Ghyslain PARENT et France BEAUMIER, «Un spectacle de danse contemporaine... ", op. cit., p. 59-73).

102. Louise BEAUDOIN, «Un projet toujours vivant: la souveraineté. Quand le Québec s’interroge», Le Monde diplomatique, février 2008, p. 29-30.

103. Pavlina HAdjitheodoulou-Loizidou et Loizos Symeou, "Promoting closer ties and cooperation between the school, the family and the community in the framework of intercultural education ", International Journal about Parents in education, vol. 1, n 0, 2007, p. 64.

104. Louise BEAUDOIN, "Quand le Québec s’interroge...", op. cit., p. 30.

105. Ibidem.

106. Sangeeta BAGGA-GUPTA, «Explorations in Bilingual Instructional Interaction... », op. cit., p. 558.

107. Marguerite BLAIS et Jules DESROSIERS, Quand les Sourds nous font signe..., op. cit.

108. Michel LELIĖVRE et Colette DUBUISSON, "Implanter une approche... », op. cit, p. 65.

109. Dominique Pinsonneault et Martin BERGEVIN, Enquête sur la formation et l'emploi..., op. cit., p. $181-182$. 


\section{CONCLUSION}

Cette étude permet d'illustrer la réalité de personnes sourdes qui ont vécu différents contextes de prise en charge éducative, allant de l'institutionnalisation (Léonard, Catherine), à l'intégration en classe d'adaptation scolaire (Amélien, Gabriel, Maxens, Jacques), à l'inclusion en milieu ordinaire (Amandine). Il faut remarquer qu'aucun des participants n'a fait d'études postsecondaires, bien qu'Amandine soit la seule à avoir tenté d'obtenir un diplôme d'études professionnelles.

Lapprentissage $d u$ français oral et écrit comme première langue joue un rôle décisif dans la décision d'arrêter, d'interrompre ou de ne pas poursuivre les études secondaires. Les témoignages des participants sourds illustrent bien le rôle de la résilience devant les obstacles rencontrés, tels que l'obtention de services d'interprétariat en LSQ. Il s'agit d'une résilience qui peut se transformer en oppression ou encore en résignation. Un tel constat nécessite selon nous d'adopter une approche d'enseignement bilingue et biculturel en contexte inclusif, propice à la libération de la personne sourde.

Les participants sourds rapportent presque tous des difficultés qui ont pu mettre à l'épreuve leur résilience dans le monde du travail, ce qui a pu se traduire sous forme d'oppression ou de résignation. La sensibilisation du milieu à la culture sourde et d'une communication adaptée, par l'accès à des services d'interprétariat, par exemple, sont des facteurs qui favorisent la libération de la personne sourde. Plusieurs participants sourds ont ainsi pu se réaliser dans des emplois de la formation professionnelle.

Des facteurs propres au microenvironnement personnel, au mésoenvironnement communautaire et au macroenvironnement sociétal facilitent le développement de la littératie et de la participation sociale de la personne sourde dans les domaines de l'éducation et du travail. Sur le plan du microenvironnement personnel, des membres de la famille ont pu jouer un rôle de facilitateur, les parents entre autres, en ayant un style défenseur, ou encore en apportant du soutien au développement de la littératie et de la participation sociale du jeune. Dans le mésoenvironnement communautaire, la direction d'établissement, les orthopédagogues, les orthophonistes, des amis, l'employeur, un collègue, un ami, peuvent également être des facilitateurs. Dans le macroenvironnement sociétal, la difficulté à reconnaître la LSQ a des répercussions sur le contexte d'organisation des services, auprès des commissions scolaires, ou encore des services externes d'aide à la main d'œuvre.

Il faut souligner l'importance qu'il y a à favoriser un contexte d'inclusion scolaire, sociale et professionnelle qui tienne compte d'une approche culturelle propre au bilinguisme et à la culture sourde. Une priorité semble 
être la reconnaissance de la LSQ non seulement de manière constitutionnelle, mais aussi comme première langue d'enseignement. Cette reconnaissance s'intégrerait à la politique interculturelle québécoise ${ }^{110}$ car elle se présente comme une nécessité si l'on veut favoriser l'intégration et la participation des personnes sourdes à la société.

110. Louise BEAUdOIN, "Quand le Québec s'interroge...", op. cit, p. 30 ; et Gérard BouCHARD et Charles TAYLOR, "Fonder l'avenir. Le temps de la conciliation", Rapport de la Commission de consultation sur les pratiques d'accommodement reliées aux différences culturelles, Québec, 2008, p. 19. 